\title{
UNIVERSITYOF BIRMINGHAM

\section{Transcutaneous electrical nerve stimulation using an LTP-like repetitive stimulation protocol for patients with upper limb complex regional pain syndrome: A feasibility study}

Ryan, Cormac; King, Rick; Robinson, Victoria; Punt, Timothy; Dinse, Hubert; Grüneberg, Christian; Johnson, Mark; Martin, Denis

DOI:

$10.1177 / 1758998316678588$

License:

None: All rights reserved

Document Version

Peer reviewed version

Citation for published version (Harvard):

Ryan, C, King, R, Robinson, V, Punt, T, Dinse, H, Grüneberg, C, Johnson, M \& Martin, D 2017, 'Transcutaneous electrical nerve stimulation using an LTP-like repetitive stimulation protocol for patients with upper limb complex regional pain syndrome: A feasibility study', Hand Therapy, vol. 22, no. 2, pp. 52-63.

https://doi.org/10.1177/1758998316678588

Link to publication on Research at Birmingham portal

\begin{abstract}
General rights
Unless a licence is specified above, all rights (including copyright and moral rights) in this document are retained by the authors and/or the copyright holders. The express permission of the copyright holder must be obtained for any use of this material other than for purposes permitted by law.
\end{abstract}

- Users may freely distribute the URL that is used to identify this publication.

- Users may download and/or print one copy of the publication from the University of Birmingham research portal for the purpose of private study or non-commercial research.

- User may use extracts from the document in line with the concept of 'fair dealing' under the Copyright, Designs and Patents Act 1988 (?)

- Users may not further distribute the material nor use it for the purposes of commercial gain.

Where a licence is displayed above, please note the terms and conditions of the licence govern your use of this document.

When citing, please reference the published version.

Take down policy

While the University of Birmingham exercises care and attention in making items available there are rare occasions when an item has been uploaded in error or has been deemed to be commercially or otherwise sensitive.

If you believe that this is the case for this document, please contact UBIRA@lists.bham.ac.uk providing details and we will remove access to the work immediately and investigate. 
Title: TENS using a LTP-like repetitive stimulation protocol for patients with upper limb complex regional pain syndrome: a feasibility study.

Authors: Ryan $\mathrm{CG}^{1}$, King $\mathrm{R}^{2}$, Robinson $\mathrm{V}^{2}$, Punt $\mathrm{DT}^{4}$, Dinse $\mathrm{H}^{6}$, Grunenberg $\mathrm{C}^{5}$, Johnson $\mathrm{MI}^{3}$, Martin $\mathrm{DJ}^{1}$.

Affiliations:

1. Institute of Health and Social Care, Teesside University, UK

2. Pain Clinic, James Cook University Hospital, South Tees Hospitals NHS Foundation Trust, UK

3. Faculty of Health and Social Sciences, Leeds Beckett University, City Campus, Leeds, UK

4. Birmingham University, UK

5. Hochschule für Gesundheit Bochum, Universitätsstrasse 105, Germany

6. Inst. Neuroinformatik, Neural Plasticity Lab, Ruhr-University Bochum

Corresponding Author

Dr Cormac Ryan PhD

Reader in Physiotherapy

Health and Social Care Institute 
Teesside University

Middlesbrough, UK.

TS1 3BA

Telephone: 0044 (0)1642 738253

Email: c.ryan@tees.ac.uk

\begin{abstract}
Introduction: This feasibility study aimed to 1) develop a clinical protocol using a LTP-like (long term potentiation) repetitive stimulation protocol for transcutaneous electrical nerve stimulation (TENS) in patients with upper limb complex regional pain syndrome (CRPS) and 2) develop a research protocol for a single-blind randomised controlled trial (RCT) investigating the efficacy of TENS for CRPS.

Methods: This small-scale single-blind feasibility RCT planned to randomise 30 patients with upper limb CRPS to either a variant of TENS or placebo TENS for 3 weeks. Stimulation comprised 20 pulses over 1-second with a non-stimulation interval of 5-seconds, a so-called repetitive electrical stimulation protocol following the timing of LTP. Pain, function and body image were measured at baseline, post-treatment and 3-months follow-up. At 3-months, participants were invited to one-to-one interviews, which were analysed thematically.
\end{abstract}


Results: A TENS protocol with electrodes applied proximal to the area of allodynia in the region of the upper arm was developed. Participant concordance with the protocol was high. The sample size was small (TENS $(n=6)$, placebo $(n=2))$. Mean (SD) pain intensity for the TENS group on a 0-10 scale was 7.2 (2.4), 6.6 (2.8), and 7.8 (1.9), at baseline, post-treatment and 3-month follow-up respectively. Qualitative data suggested some patients found TENS beneficial, easy to use, and were still using at 3-months. Discussion: Patients tolerated TENS well and important methodological information to facilitate the design of a large-scale trial was obtained. [ISRCTN48768534]

Key words: Complex regional pain syndrome; TENS; feasibility study; upper limb 


\section{Introduction}

Transcutaneous electrical nerve stimulation (TENS) is commonly used for pain relief, has few side-effects and can be self-administered using relatively inexpensive devices. ${ }^{1}$ Recent guidelines ${ }^{2}$ identify TENS as a potential therapy for patients with Complex Regional Pain Syndrome (CRPS). However no randomised controlled trials (RCTs) have investigated the effectiveness of TENS for CRPS.

The primary mechanism by which TENS purports to work is via the pain gate. In brief, TENS activates large diameter, low threshold mechanosensitive afferents resulting in synaptic inhibition of nociceptive transmission in the spinal cord and brainstem. ${ }^{3} \mathrm{We}$ propose that TENS may have additional mechanisms of action for painful conditions that involve perceptual distortions of the affected area.

Persistent pain can disrupt the representation of the painful body part in the brain of CRPS patients, often referred to as cortical reorganisation/disruption. This cortical reorganisation is hypothesised to play a role in maintaining the pain. ${ }^{4,5}$ Sensory retraining can be defined as any intervention aimed at restoring the normal body image of that area. Evidence from patients with phantom limb pain and CRPS suggest that sensory retraining of the painful body part, by physical stimulation of that body part, can normalise the disrupted body image and reduce pain. ${ }^{6-8}$ TENS, delivered in a 
synchronised pulsed fashion across two or more receptive fields, commonly referred to as repetitive electrical stimulation, has been used as a method of sensory retraining in individuals with diminished sensorimotor performance, such as older adults and stroke patients. ${ }^{9-12}$ Additionally, a recent non-randomised study using high-frequency repetitive sensory (LTP)-like (long term potentiation) stimulation suggested it could reduce sensory impairment in patients with CRPS. ${ }^{13}$ To date no RCTs have investigated the effect of TENS on sensorimotor performance in patients with CRPS. However, it has been shown that TENS relieves phantom pain and facilitates perceptual embodiment of prosthetic limbs ${ }^{14}$ when TENS sensation is projected into the phantom limb itself. ${ }^{15}$ We have hypothesised that TENS may be mimicking normal neural input to restore disrupted body image. ${ }^{16}$

There is a need for a full scale blinded RCT to investigate the efficacy of TENS to reduce pain and improve sensorimotor function in patients with CRPS. However, beforehand a feasibility study is warranted to ensure the methodological components of the protocol are fit for purpose. ${ }^{17}$ Many full-scale trials fail because a feasibility study was not undertaken. ${ }^{18}$ Thus the purpose of this feasibility study was to 1 ) develop a clinical protocol using a LTP-like repetitive stimulation protocol for TENS in patients with CRPS and 2) develop a research protocol for a single-blind RCT investigating the 
efficacy of TENS for CRPS. To achieve these aims this study had a number of objectives (see table 1).

Insert table 1

\section{Method}

\section{$\underline{\text { Study overview }}$}

This mixed-methods single-blind placebo controlled feasibility study randomised participants with CRPS into an intervention or a placebo group. The aims were to 1) develop a clinical protocol using a LTP-like repetitive stimulation protocol for TENS in patients with CRPS and 2) develop a research protocol for a single-blind RCT investigating the efficacy of TENS for CRPS.

\section{$\underline{\text { Participants, recruitment, screening and randomisation }}$}

The study aimed to recruit 30 participants from a regional NHS hospital in the north east of England. This study was approved by the NRES Committee North East - York NHS Ethics Committee (REC approval number: 13/NE/0286). Participants were eligible for inclusion if they were $\geq 18$ years of age and had type-1 CRPS of the upper limb defined by the Budapest criteria ${ }^{19}$ for $\geq 6$ months' duration. Those who once fulfilled the criteria, no longer did, but had ongoing pain were classed as CRPS-NOS [not otherwise specified], and were also included. Participants were excluded if they 
found TENS as applied in the $1^{\text {st }}$ session unacceptable/intolerable, lacked the capacity to give informed consent, had any neurological condition (e.g. stroke) or were unable to speak English. Eligible participants provided informed consent and were randomly allocated to TENS or placebo TENS using a random number generator and pre-filled concealed envelopes by a team member uninvolved in participant contact. As this was a feasibility study an apriori sample size calculation was not undertaken though the sample size was in keeping with recommendations that such trials should aim for approximately $n=12$ in each group. ${ }^{20}$ This study aimed to recruit 30 participants which would allow for a $20 \%$ drop out rate whilst still achieving $n=12$ per group.

\section{$\underline{\text { Preliminary rehearsal of process }}$}

Based upon our previous clinical experience and direct patient involvement in the study design, it was felt that CRPS patients were unlikely to tolerate applying TENS electrodes to the painful site. Thus, we proposed TENS be applied to the upper arm, proximal to the affected site and the TENS sensation projected distally into the affected area. We easily achieved this distal projection in unimpaired participants (unpublished). This simple adaption of conventional TENS technique (e.g. placing the electrodes over the painful area) overcame this unique CRPS barrier.

\section{Intervention}


TENS, in this study, was delivered using a commercially available two-channel TENS unit (Elpha II 3000 muscle and pain stimulator, Danmeter). A detailed demonstration on self-application and a simple instruction sheet to facilitate home use was provided. Pulsed, synchronised dual channel TENS was self-administered (for 90 minutes) at home, daily, over a period of 3 weeks. The stimulation pattern described by Freyer et al. (2012) $)^{12}$ was used: 20 pulses delivered over a 1-second period (20Hz stimulation frequency) with a non-stimulation interval of 5-seconds. This form of stimulation is commonly referred to as repetitive electric stimulation and its primary proposed mechanism of action is cortical reorganisation rather than via the classic gate control mechanism associated with conventional TENS stimulation. A self-report diary monitored home programme concordance. All participants received usual care provided by the same physiotherapist to maximise standardisation. Usual care physiotherapy included but was not limited to; advice, education, exercise, Cognitive Behavioural Therapy, motor imagery, hand laterality recognition training, desensitizing and hydrotherapy. It excluded TENS or other electrotherapy modalities. The number of usual care physiotherapy sessions was recorded for all participants.

\section{$\underline{\text { Placebo }}$}

The Placebo group received TENS in an identical manner to the active intervention except that no electrical current was delivered, although the power light flashed 
pulsatingly indicating that the device was switched on. It was impossible to blind participants to receiving a strong non-painful TENS sensation. However, we attempted to markedly reduce this bias by introducing uncertainty as to whether a strong sensation was a pre-requisite for pain relief. Hence, information orientating participants to study aims and procedures were designed to raise uncertainty in the mind of the participant about whether they received an active intervention or not. This approach has been used successfully in previous studies. ${ }^{21}$ Blinding the therapist was not possible. Similar to the participants in the intervention group, all participants in the placebo group received usual care provided by the same physiotherapist and the number of usual care physiotherapy sessions was recorded for all participants.

\section{$\underline{\text { Clinical Outcomes }}$}

Pain: Pain was measured using a Visual Analogue Scale (VAS) with the question "How would you rate your average pain over the last two days?” and anchor statements of "no pain” and “worst imaginable pain”. The VAS is valid and reliable. ${ }^{22}$ The VAS data was collected at baseline; post treatment and 3-month follow-up. A change of $\geq 30 \%$ was considered clinically meaningful. ${ }^{23}$ As pain was considered the primary outcome measure, the effect size was also calculated for this outcome and used to calculate the sample size for a future fully powered trial. Effect size was calculated as the change from pre to post treatment divided by the standard deviation of the baseline values. ${ }^{24}$ 
Pain Diary: Participants also kept a pain diary rating their daily pain (0-10 four times (Morning, afternoon, evening and night) using the following question: "How would you rate your pain at this point in time?” and anchor statements of "no pain” and "worst imaginable pain”.

Function: Function was assessed using the Disabilities of the Arm, Shoulder and Hand test (DASH) a 30-item questionnaire that is scaled from 0-100. The DASH has a good level of validity, reliability and sensitivity to change. ${ }^{25}$ A change of $\geq 15$ points was considered a meaningful improvement ${ }^{26}$.

Pain Medication Use: Daily medication use was recorded in a diary for each day capturing if pain medication was used, and if so what type and dosage (number of pills \& size (mg) of pills). ${ }^{27}$

Adverse reactions: If any participant reported an adverse reaction this was recorded.

\section{$\underline{\text { Perceptual Outcomes }}$}

Hand Laterality Recognition Task: Correctly identifying the laterality (i.e. 'left' or 'right') of an image depicting a hand requires the participant to engage in the mental 
rotation (motor imagery) of their own limbs, ${ }^{28}$ with optimal performance relying on an intact body schema. Previous studies have demonstrated impairment on the task in patients with CRPS. ${ }^{29-32}$ Participants were presented with images of hands on a computer screen of varying laterality, view, orientation and rotation; and asked to respond as quickly and as accurately as possible. Presentation of stimuli (images) and resulting data (accuracy and response time) were controlled and logged using customized software (E-Prime, www.pstnet.com).

Bath CRPS Body Perception Disturbances: The Body Perception Disturbance questionnaire assesses a patients’ perception of their affected limb. ${ }^{33}$ Participants rate different aspects of perception such as feelings of ownership and attention. Five of the items are on a $0-10$ scale, 2 of the items are yes/no answers and the final item is graded on a $0-1-2$ scale. A total score is scaled from $0-57$. Higher scores denote poorer perception of the limb. There is no literature identifying what is a meaningful clinical improvement on the Body Perception Disturbance questionnaire.

\section{$\underline{\text { Research outcomes }}$}

Expectation and Blinding: To assess patient expectation at the beginning of the study participants were asked to rate the following: A) How helpful do you believe TENS will be for your pain?: 0 = not at all helpful 10 = Extremely helpful; B) how helpful do you 
believe sub threshold TENS will be for your pain?: $0=$ not at all helpful $10=$ Extremely helpful; C) How easy to use do you think TENS will be?: $0=$ not at all easy to use $10=$ extremely easy to use. ${ }^{34}$

To investigate the appropriateness of the single-blinding procedures, participants were asked to make a judgement as to which group they were randomised. The blinding assessment method asked the participants two questions. Firstly, “do you believe your TENS unit was functioning properly?”. The five possible responses to were: 1) I am certain my TENS unit was working properly; 2) I think my TENS unit was probably working properly; 3) I have absolutely no idea whether the TENS unit was working properly or not; 4) I think my TENS unit was probably not working properly; 5) I am certain my TENS unit was not working properly. Secondly, "If above you answered properly 3) I have absolutely no idea whether the TENS unit was working properly or not - what would you guess 1) functioning properly 2) no functioning properly”. This assessment method has been previously used by our group and others in the TENS field. ${ }^{35}$

Recruitment/eligibility: The number of participants recruited over 20 months was used to judge the recruitment rate. Additionally the number of participants invited to take part but did not meet the eligibility criteria on formal screening were recorded, to assess 
the eligibility criteria. Participants were invited to provide a reason for not enrolling if they wished to do so.

\section{Qualitative Interviews}

All participants were invited to undertake an exit interview at the 3-month point to gather patient's perceptions of the intervention. The brief semi-structured interviews ( $<10$ minutes) were audio-recorded and typed verbatim. The interview topic guide is shown in table 2. The qualitative methodology used was based on interpretative phenomenological analysis (IPA) as described by Smith and Osborn (1998). ${ }^{36}$ IPA is a useful approach for gaining insight into patient experience of an intervention. The interviews were undertaken by RK and the primary analysis was undertaken by CR. The primary analysis involved the reading and re-reading of transcripts. Notes were made in the transcript margins to facilitate the development of emergent themes. The analysis was provided to a $2^{\text {nd }}$ member of the team (DM) who read all the transcripts and confirmed that the emergent themes were rooted in the data. ${ }^{36}$ Discussion occurred between the CR and DM to develop the final themes that are presented. Participant quotes were used to support the theme development.

Insert table 2 


\section{$\underline{\text { Data analysis }}$}

Participant characteristics are presented as mean and 1SD. As this was a feasibility study, the change in outcomes were investigated using descriptive statistics. The qualitative data were analysed thematically.

\section{Results}

\section{$\underline{\text { Recruitment/eligibility }}$}

Thirteen individuals initially showed interest in participating of whom eight consented and were randomised to the TENS $(n=6)$ or placebo TENS group (n=2) (Figure 1). There was a 50\% drop-out rate with only four participants providing data at the 3 month follow-up point. The participant characteristics are shown in table 3. The number of usual care physiotherapy sessions received by each participant ranged from 0-14 (table $3)$.

Insert figure 1 and table 3

\section{$\underline{\text { Clinical outcomes }}$}

Pain: Participants’ outcomes for pain are shown in Figure 2A-C. Only Participant 003 in the TENS group showed a meaningful improvement post intervention (31\% improvement post treatment) but not at the three month follow-up (22\%). Group mean 
(SD) pain intensity for the TENS group was 7.2 (2.4), 6.6 (2.8), and 7.8 (1.9), at baseline, post-treatment and 3-month follow-up, respectively. Thus, the effect size from pre to post treatment was 0.25 . For the single placebo group participant (006) with post/follow-up data there was a meaningful improvement in pain post-treatment and at 3 month follow up (74\% and 71\%, respectively).

Pain Diary: Only four of the participants used the pain diary. Of these, one participant used it for two weeks while the other three used it for all three weeks. The daily pain levels and device usage data show no obvious trend between groups (See figure 4A-B).

Pain medication usage: The pain medication usage diary was sparsely completed and provided little or no useful or coherent information.

Function: Participants’ outcomes for function are shown in Figure 2A-C. Only Participant 004 in the TENS group showed a meaningful improvement (24 points at three month follow-up). Group mean (SD) DASH scores for the TENS group were 66 (21), 75 (15), and 67 (30), at baseline, post-treatment and 3-month follow-up, respectively. For the single placebo group participant (participant 006), who had post/follow-up data, there was no meaningful change in pain.

Adverse Reactions: None of the participants formally reported an adverse reaction. However, during their exit interview, one participant in the placebo group reported experiencing a short-term mild increase in hand swelling. 


\section{$\underline{\text { Perceptual outcomes }}$}

Hand Laterality Recognition Task ${ }^{28}$ : Participants’ outcomes for hand laterality recognition are shown in Figure 3A-D. Inspection of raw data suggests a gradual reduction in reaction time in both groups but there was no obvious pattern within the accuracy data.

Bath CRPS Body Perception Disturbance ${ }^{33}$ : Participants' outcomes for perceived body perception are shown in Figure 2A-C. Higher scores denote poorer perception of the limb. Group mean (SD) Body Perception Disturbance scores for the TENS group were 30 (16), 33 (18), and 33 (21), at baseline, post-treatment and 3-month follow-up, respectively. For the single placebo group participant (006) with post/follow-up data Body Perception Disturbance scores were 14, 11, and 8, at baseline, post-treatment and 3-month follow-up, respectively.

\section{$\underline{\text { Patient expectations and blinding }}$}

At baseline both the TENS and the placebo groups had similar levels of expectations regarding how helpful they believed TENS will be for their pain [5.8 (2.7) vs. $6.5(0.0)]$, how helpful they believed sub-threshold TENS would be for their pain [5.2 (2.9) vs. 5.0 (2.1)], and how easy they thought TENS would be to use [6.4 (3.5) vs. 5.8 (5.4)].

At the 3-month follow-up all three of the participants from the TENS group who completed these questions were certain that their TENS unit was working correctly 
while the one participant from the placebo group thought that the TENS unit was working correctly but was not certain.

\section{Qualitative Interviews}

Four ( $n=3$ TENS and $n=1$ placebo) participants underwent exit interviews. Two main themes were identified: 1) level of perceived benefit and 2) ease of use.

Theme 1:Level of perceived benefit

Of the three TENS participants, one clearly stated that they did not find TENS useful: “[TENS] didn't work” [002]

The other two TENS participants found TENS beneficial. Participant 003 stated that when the TENS was being applied it helped to take her mind off her pain and reported relief of about $50 \%$ when TENS was on but no residual benefit once removed:

"It took my mind away from the pain while it was on...I would say about 50\% [reduction in pain] " [003]

Both of these participants were still regularly using TENS (three or four times a day [004]) but were now using it in a continuous mode rather than the pulsed mode that was used in the study:

"I did [get my own TENS machine] yeah and I keep that on the full one [continuous mode]" [003] 
There was no obvious reason identified as to why some participants found TENS beneficial and some didn’t. However, the participant reporting no benefit described feeling the TENS sensation in their arm/elbow while their main painful area was their thumb. In contrast one of the participants who found a benefit specifically reported feeling the TENS sensation in the same area as their pain.

The single participant in the placebo group who undertook an exit interview also felt that the TENS had been beneficial though, they qualified that the benefit was small and felt that the effect was due to distraction.

"I think it did decrease pain but only minimally, and I think that was possibly more due to distraction rather than actually the TENS" [006]

This participant noted a short-term adverse event. The participant self-managed this adverse event. They did not formally report it as an adverse event within the study itself.

"A slight increase in swelling in my hand...could be combatted with the compression glove” [006]

Theme 2: Ease of use

All four participants reported that TENS was easy to use.

“very easy to use” [002] 
While, the application of the electrode pads and their associated wires did pose challenges patients learned to problem solve the issues independently or with the help of a partner.

"I struggled at first because the wires getting in and out, obviously I can't pull the wires totally out because it's my right hand...but I have worked it out now I pull the pads off...or I just leave them plugged in and my partner takes them out” [004]

“Other than the fact that I can't put the pads on my shoulder myself, it's completely usable” [006]

Insert figure $2 A-C$

Insert figure $3 A-D$

Insert figure $4 A-B$

\section{Discussion}

We developed a clinical TENS protocol where the electrode pads were applied to the upper arm, proximal to the affected site and the TENS sensation projected distally down the arm into the affected area. This simple adaption of conventional TENS technique (e.g. placing the electrodes over the painful area) was acceptable to participants who 
reported during interview that the device was easy to use and had no adverse effects apart from a short-term mild increase in hand swelling in one participant in the placebo group. Participant concordance to the treatment regime was high implying that the application technique was acceptable. In addition this feasibility study identified key methodological issues, such as pain medication usage recording, which should be taken into consideration for a future RCT.

Recruitment rates were poor. This does not appear to have been the result of inappropriate recruitment procedures per se or overly exclusive eligibility criteria. When planning this study it was estimated that the pain department hosting the study usually had 3 new patients with CRPS per month, with 2 new patients per week attending local peripheral clinics. Thus, it was expected that recruitment goals were achievable. A number of strategies were put in place to increase recruitment including regular updates to staff to raise awareness of the study; however, it is unclear what if any effect such strategies had. During the recruitment phase other clinical studies/clinical pathways were operating within the hospital, which may have reduced the number of individuals directed towards this study. A future large scale RCT should be multicentred and consider using existing databases such as the UK CRPS database. ${ }^{37}$

The pain and TENS usage diary were useful outcome measures though only four of the eight participants completed them. The reason for this poor completion is unknown. 
Those participants who completed diaries did so comprehensively with little missing data. The TENS usage data indicated a high level of treatment concordance with usage above the recommended 90 minutes in 64 of the 69 reported usage days (93\% adherence). Assuming zero usage on the days that no information was inputted by the four participants this would still indicate $76 \%$ concordance (64/84). This high usage occurred alongside relatively unchanging pain levels. Pain medication recording was inconsistent and ultimately the information provided was difficult to interpret and thus unusable. Other methods of monitoring pain medication usage, such as automated tracking $^{38}$ should be explored. In addition, monitoring the number of usual care physiotherapy sessions was an important step with respect to monitoring cointerventions. The number of sessions varied quite considerably between participants which may need to be adjusted for in a future fully powered trial.

There were significant challenges with both of the perceptual outcomes: the Hand Laterality Recognition Task ${ }^{28}$ and Body Perception Disturbance questionnaire. ${ }^{33}$ Due to technical difficulties much of the Hand Laterality Recognition Task data was not collected. The mean (SD) Body Perception Disturbance score for all participants was 28 (15), which is higher than previously reported values for CRPS patients (21 (11)). ${ }^{39}$ The Body Perception Disturbance scores showed no obvious pattern with scores remaining largely unchanged, and the absence of any existing data regarding minimally important 
difference makes it difficult to comment upon the importance of the small changes seen. The Body Perception Disturbance questionnaire contains a component where the patient describes their mental image of their affected limb and this is drawn by the assessor/health care professional. This drawing influences the scoring of the questionnaire. The clinician reported that the drawing was heavily influenced by their artistic ability rather than the patient's description, questioning the face validity of the tool. A complete assessment of the psychometric properties of the Body Perception Disturbance has not been undertaken as yet ${ }^{39}$ but future work may want to consider the utility of the drawing component.

The level of expectation in participants regarding both above threshold and sub threshold TENS was similar between groups suggesting our approach to make the no current placebo TENS, a viable intervention in the eyes of the participants was successful. However, regarding blinding at the 3-month follow-up there appeared to be an element of doubt about the function of the device in the placebo group that was not apparent in the TENS group implying that the blinding of participants may not have been completely successful, though it does imply a degree of success. However it is difficult to determine from the limited amount of data. 
Within the qualitative interviews, one participant stated that TENS was of no benefit to them, this could have been related to the difficulty in projecting the TENS sensation over the painful thumb area for this patient. The other two participants who provided interviews in the TENS group both reported finding TENS beneficial. One reported a clinically relevant $50 \%$ reduction in pain when using TENS and both were still using TENS regularly at the 3-month point. Interestingly they choose to use the continuous mode of delivery compared to the burst mode used for repetitive electrical stimulation as used in this study. Furthermore, one of the participants emphasised that TENS was beneficial during stimulation but effects were short-lived once the TENS device had been switched off. It has been argued that TENS pain relief should only be expected when in situ and thus outcome measurement to test its effectiveness should occur at this point. $^{40}$

\section{$\underline{\text { Study implications }}$}

The implications of this study is that this trial does not, as yet, appear feasible at a definitive trial level in its current form. Recruitment is a major issue and would need to be addressed in further feasibility work before proceeding to a full trial. In addition, data collection processes need to be further enhanced, with particular emphasis on the pain medication usage diary, the body perception questionnaire ${ }^{33}$ and the Hand Laterality Recognition Task ${ }^{28}$. One objective of this work was to calculate an effect size to inform 
a fully powered future trial. For the primary outcome measure of pain, from pre- to post-treatment an effect size of 0.25 was shown. Using this effect size, an alpha level of 0.05, and $80 \%$ power, a sample size of 506 participants (253 per group) would be required for a fully powered trial. Given the difficulties with recruitment shown in this study such a sample size would likely be very difficult to attain.

\section{$\underline{\text { Study limitations }}$}

This study was limited by a number of factors already discussed above, not least the low recruitment rates and the relatively high dropout rates (50\%). We did not obtain reasons for dropout, this should be explored in future work. The imbalance in the randomisation was also an issue. The randomisation had been undertaken to ensure an equal number of 15 participants would be randomised to each group, however, by chance the random sequence initially was loaded towards assigning more to the intervention group rather than the placebo group. Blocked randomisation would have prevented this distortion and should be used in any future small-scale trials. In addition there was limited investigation of fidelity within the study, beyond the monitoring the concordance to the recommended usage dose (90 minutes daily) via a diary. Further checks of the quality of delivery of the intervention in the participant's home environment would have been useful. 


\section{Conclusion}

We developed a clinical TENS protocol where the electrode pads were applied to the upper arm, proximal to the affected site and the TENS sensation projected distally down the arm into the affected area. Participants tolerated TENS well. A number of methodological issues were identified, such as low recruitment rates and inadequate data collection processes particularly regarding pain medication usage and body perception assessment. Thus, further feasibility work across multiple sites, focussing upon these methodological issues is warranted prior to undertaking a full scale RCT.

\section{Declaration of conflicting interests}

The authors declare that they have no conflicts of interest.

\section{References}

1. Johnson MI. The Role of Transcutaneous Electrical Nerve Stimulation (TENS) in Pain Management. In: Colvin LA (eds) ABC of Pain. BMJ Books: Blackwell Publishing, 2012, pp.91-98. 
2. Goebel A, Barker $\mathrm{CH}$, Turner-Stokes L, et al. Complex regional pain syndrome in adults: UK guidelines for diagnosis, referral and management in primary and secondary care. London: RCP, 2012.

3. Johnson MI and Bjordal JM. Transcutaneous electrical nerve stimulation for the management of painful conditions; focus on neuropathic pain. Expert Rev Neurother 2011; 11: 735-753.

4. Pleger B, Tegenthoff M, Ragert P, et al. Sensorimotor Returning in Complex Regional Pain Syndrome Parallels Pain Reduction. Ann Neurol 2005; 57: 425429.

5. Pleger B, Ragert P, Schwenkreis $\mathrm{P}$, et al. Patterns of cortical reorganization parallel impaired tactile discrimination and pain intensity in complex regional pain syndrome. Neuroimage 2006; 32: 503-510.

6. Flor H, Denke C, Schaefer M, et al. Effect of sensory discrimination training on cortical reorganisation and phantom limb pain. Lancet 2001; 357: 1763-1764.

7. Moseley GL, Zalucki NM and Wiech K. Tactile discrimination, but not tactile stimulation alone, reduces chronic limb pain. Pain 2008; 137: 600-608.

8. Moseley GL and Weich K. The effect of tactile discrimination training is enhanced when patients watch the reflected image of their unaffected limb during training. Pain 2009; 144: 314-319. 
9. Smith PS, Dinse HR, Kalisch T, et al. Effects of Repetitive Electrical Stimulation to Treat Sensory Loss in Persons Poststroke. Arch Phys Med Rehabil 2009; 90: 2108-2111.

10. Kalisch T, Tegenthoff M and Dinse HR. Repetitive Electric Stimulation Elicits Enduring Improvement of Sensorimotor Performance in Seniors. Neural Plasticity 2010; 2010.

11. Kattenstroth J, Kalisch T, Peters S, et al. Long-term sensory stimulation therapy improves hand function and restores cortical responsiveness in patients with chronic cerebral lesions. Three single case studies. Frontiers in human neuroscience 2012; 6: (244): 1-13.

12. Freyer F, Reinacher M, Notle G, et al. Repetitive tactile stimulation changes resting-state functional connectivity - implications for treatment of sensorimotor decline. Frontiers in human neuroscience 2012; 6 (144) 1-11.

13. David M, Dinse HR, Mainka T, et al. High-Frequency Repetitive Sensory Stimulation as Intervention to Improve Sensory Loss in Patients with Complex Regional Pain Syndrome I. Frontiers in neurology 2015; 6: 242.

14. Mulvey MR, Fawkner HJ, Radford HE, et al. Perceptual Embodiment of Prosthetic Limbs by Transcutaneous Electrical Nerve Stimulation. Neuromodulation 2012;15: 42-47. 
15. Mulvey MR, Radford HE, Fawkner HJ, et al. Transcutaneous Electrical Nerve Stimulation for Phantom Pain and Stump Pain in Adult Amputees. Pain Practice 2012: 13(4): 289-296.

16. Mulvey MR, Fawkner HJ, Radford HE, et al. The use of transcutaneous electrical nerve stimulation (TENS) to aid perceptual embodiment of prosthetic limbs. Med Hypotheses 2009; 72: 140-142.

17. Craig P, Dieppe P, Macintyre S, et al. Developing and evaluating complex interventions: the new Medical Research Council guidance. BMJ 2008; 337: 1655.

18. Arain M, Campbell MJ, Cooper CL, et al. What is a pilot or feasibility study? A review of current practice and editorial policy. BMC medical research methodology 2010; 10: 67.

19. Harden RN, Bruehl S, Perez RS, et al. Validation of proposed diagnostic criteria (the “Budapest Criteria”) for complex regional pain syndrome. Pain 2010; 150(2): 268-274.

20. Julious SA. Sample size of 12 per group rule of thumb for a pilot study. Pharmaceutical Statistics 2005; 4(4): 287-291.

21. Francis RP, Marchant P and Johnson MI. Conventional versus acupuncture-like transcutaneous electrical nerve stimulation on cold-induced pain in healthy 
human participants: effects during stimulation. Clin Physiol Funct Imaging 2011; 31: 363-370.

22. Von Korff M, Jensen MP and Karoly P. Assessing global pain severity by selfreport in clinical and health services research. Spine 2000; 25: 3140-3151.

23. Forouzanfar, T, Weber WEJ, Kemler M, and van Kleef $M$. What is a meaningful pain reduction in patients with complex regional pain syndrome type 1 ? The Clin J Pain 2003; 19(5): 281-285.

24. Vermeulen, HM, Breedveld FC, Le Cessie S, Rozing PM, van den Ende CHM, and Vliet Vlieland TPM. Responsiveness of the shoulder function assessment scale in patients with rheumatoid arthritis. Ann Rheum Dis 2006; 65(2): 239-241.

25. Gummeson C, Atroshi I and Ekdahl C. The disabilities of the arm, shoulder and hand (DASH) outcome questionnaire: longitudinal construct validity and measuring self-rateed health change after surgery. BMC Musculoskeletal Disorders 2003; 4:11.

26. Beaton DE, Katz JN, Fossel AH, Wright JG, Tarasuk V and Bombardier C. Measuring the whole or the parts? Validity, reliability, and responsiveness of the DASH outcome measure in different regions of the upper extremity. $J$ Hand Ther 2001; 14(2): 128-142.

27. Bjordal JM, Johnson MI and Ljunggreen AE. Transcutaneous electrical nerve stimulation (TENS) can reduce postoperative analgesic consumption. A meta- 
analysis with assessment of optimal treatment parameters for postoperative pain. Eur J of Pain 2003; 7: 181-188.

28. Parsons LM. Temporal and Kinematic Properties of Motor Behavior Reflected in Mentally Simulated Action. J Exp Psychol 1994; 20: 709-730.

29. Moseley GL. Why do people with complex regional pain syndrome take longer to recognize their affected hand? Neurology 2004; 62: 2182-2186.

30. Reinersmann A, Haarmeyer GS, Blankenburg M, et al. Left is where the L is right. Significantly delayed reaction time in limb laterality recognition in both CRPS and phantom limb pain patients. Neurosci Lett 2010; 486: 240-245.

31. Schwoebel J, Coslett HB, Bradt J, et al. Pain and the body schema: Effects of pain severity on mental representations of movement. Neurology 2002; 59: 775777.

32. Schwoebel, J, Friedman R, Duda N, et al. Pain and the body schema: Evidence for the peripheral effects on mental representations of movement. Brain 2001; 124: 2098-2104.

33. Lewis J and McCabe S. Body Perception Disturbance (BPD) in CRPS. Practical Pain Management 2010; 10: 60-66.

34. Kalauokalani D, Cherkin DC, Sherman KL, et al. Lessons from a Trial of Acupuncture and Massage for Low Back Pain: Patient Expectations and Treatment Effects. Spine 2001; 26(13): 1418-1424. 
35. Deyo RA, Walsh NE, Schoenfeld LS, et al. Can Trials of Physical Treatments Be Blinded? The Example of Transcutaneous Electrical Nerve Stimulation for Chronic Pain. Am J Phys Med Rehabil 1990; 69: 6-10.

36. Osborn M and Smith J. The personal experience of chronic benign lower back pain: An interpretative phenomenology analysis, Br J Health Psychol 1998; 3: 65-83.

37. Shenker N, Goebel A, Rockett M, et al. Establishing the characteristics for patients with chronic Complex Regional Pain Syndrome: the value of the CRPSUK Registry. British journal of pain 2015; 9(2): 122-128.

38. Yarin P, Fletcher R, DiPisa J, et al. Systems and methods for monitoring patient compliance with medication regimens. Patent 6,294,999, USA, 2001.

39. Lewis JS and Schweinhardt P. Perceptions of the painful body: the relationship between body perception disturbance, pain and tactile discrimination in complex regional pain syndrome. European Journal of Pain, 2012; 16(9): 1320-1330.

40. Johnson MI. Appropriate electrode sites and electrical characteristics for TENS. In: Johnson MI Transcutaneous Electrical Nerve Stimulation (TENS). Research to Support Clinical Practice. Oxford University Press, 2014, pp.64-92. 


\section{Figure Legends}

Figure 2A-C: Data are presented for each individual participant. * indicates the participant received the placebo intervention.

Figure 3A-D: Data are presented for each individual participant. * indicates the participant received the placebo intervention.

Figure 4A-B: The top graph (A) shows the average pain daily pain reported by each participant that completed a diary. The bottom graph (B) shows the total TENS usage time each day for each participant that completed at diary. Missing data was left blank. *indicates that participant 6 was in the placebo group while participants 2, 3 and 8 were in the intervention group. 
Table 1: Study objectives

- To explore participants' perceptions of the acceptability of TENS as delivered in this study.

- To investigate participants' concordance with the protocol for home-based delivery of TENS and to explore their opinions of factors that may influence concordance.

- To record the occurrence of any adverse effects.

- To investigate recruitment procedures and rates of recruitment.

- To investigate the appropriateness of the blinding procedure.

- To select appropriate outcome measures of limb perception.

- To investigate the appropriateness of the eligibility criteria.

- To provide preliminary data on the clinical effectiveness of TENS.

- To estimate effect sizes for outcome measures to inform future sample size calculation. 
Table 2: Interview topic guide

1. What were your general impressions of the treatment?

2. What were your expectations of treatment and to what degree do you feel these expectations were met?

3. What is your opinion of the usability and acceptability of the intervention/placebo?

4. What is your opinion about using this intervention in the future?

5. Is there anything else you would like to say about the intervention/study? 
Table 3: Participant characteristics

\begin{tabular}{ccccccc}
\hline Participant & Group & Affected side & Age & Gender & TENS position & No. of Usual Care \\
& & & & & & PT sessions \\
\hline 001 & TENS & Left & 47 & Male & Elbow/wrist (median) & 4 \\
002 & TENS & Right & 50 & Female & Shoulder/elbow & 6 \\
003 & TENS & Left & 34 & Female & Elbow/wrist (median) & 7 \\
004 & TENS & Right & 32 & Female & Elbow/wrist (median) & 11 \\
005 & TENS & Left & 34 & Male & Elbow/wrist (ulnar) & 1 \\
008 & TENS & Left & 37 & Female & Elbow/wrist (median, & 5 \\
006 & Placebo & Right & 29 & Female & Elbow/wrist (median) & 14 \\
009 & Placebo & Right & 36 & Female & Hand (median) & 20 \\
\hline
\end{tabular}




\section{Figure 1: Participant flow diagram}

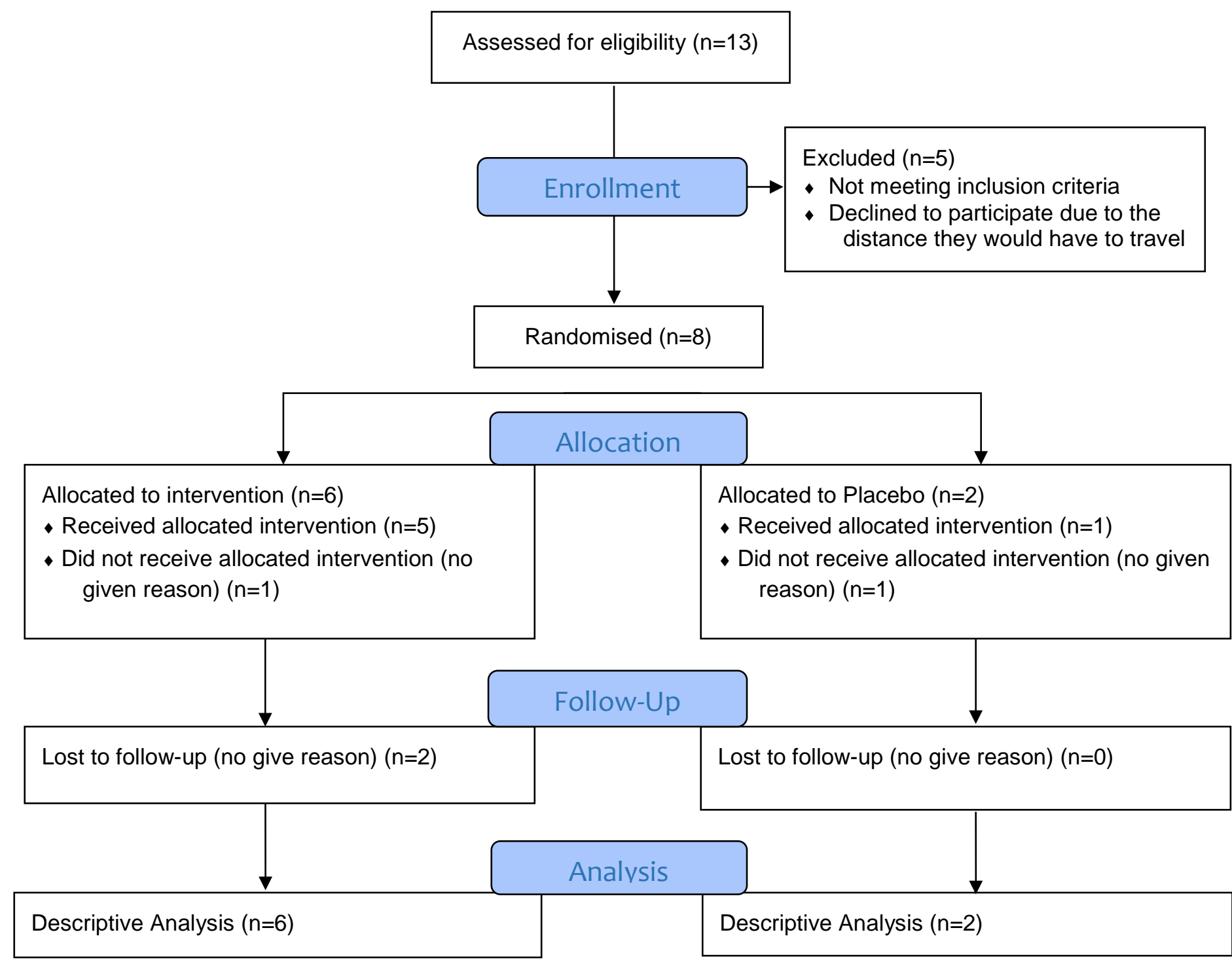


Figure 2A: Pain VAS

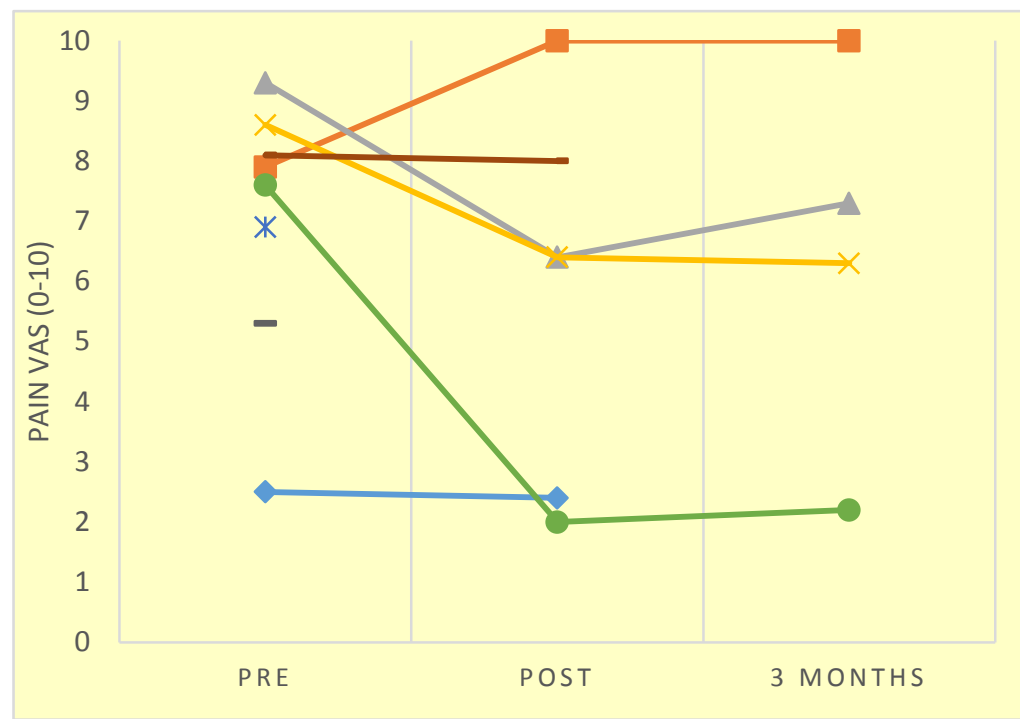

Figure 2B: Disability and Shoulder Assessment (DASH)

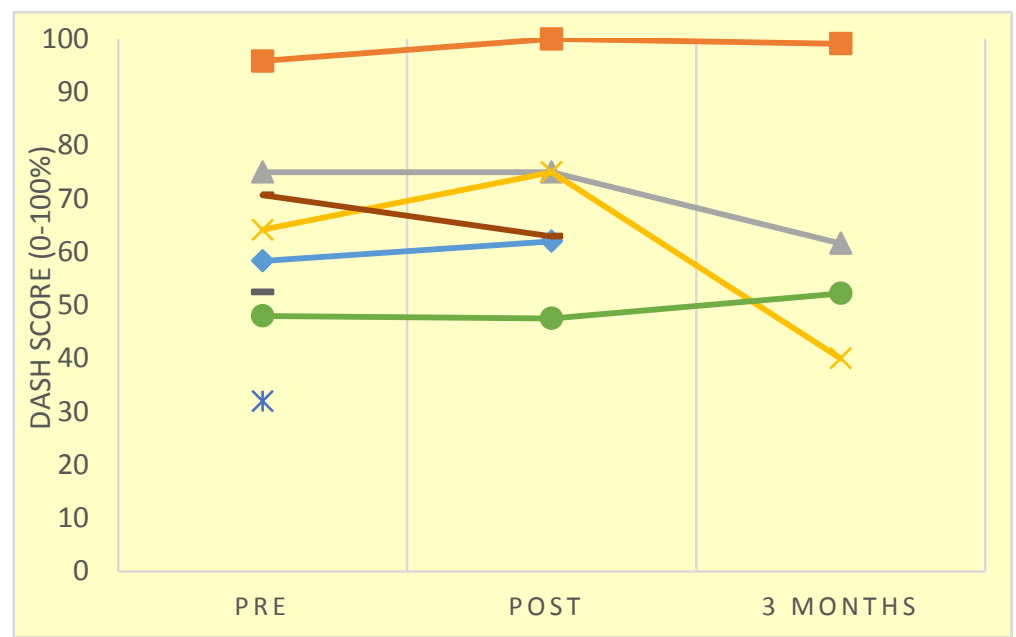

Figure 2C: Bath CRPS Body Perception Disturbances (BPD) 


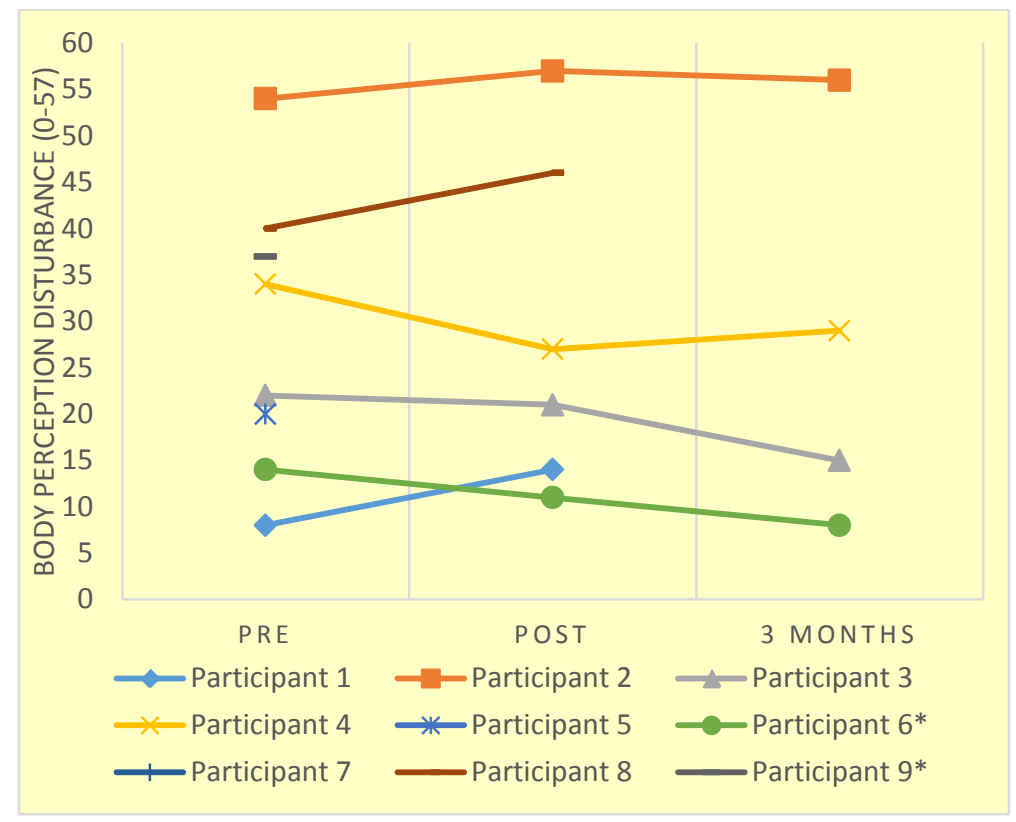

Figure Legend 2A-C: Data are presented for each individual participant. * indicates the participant received the placebo intervention. 
Figure 3A-D: Hand Laterality Recognition Task (HLRT) outcomes

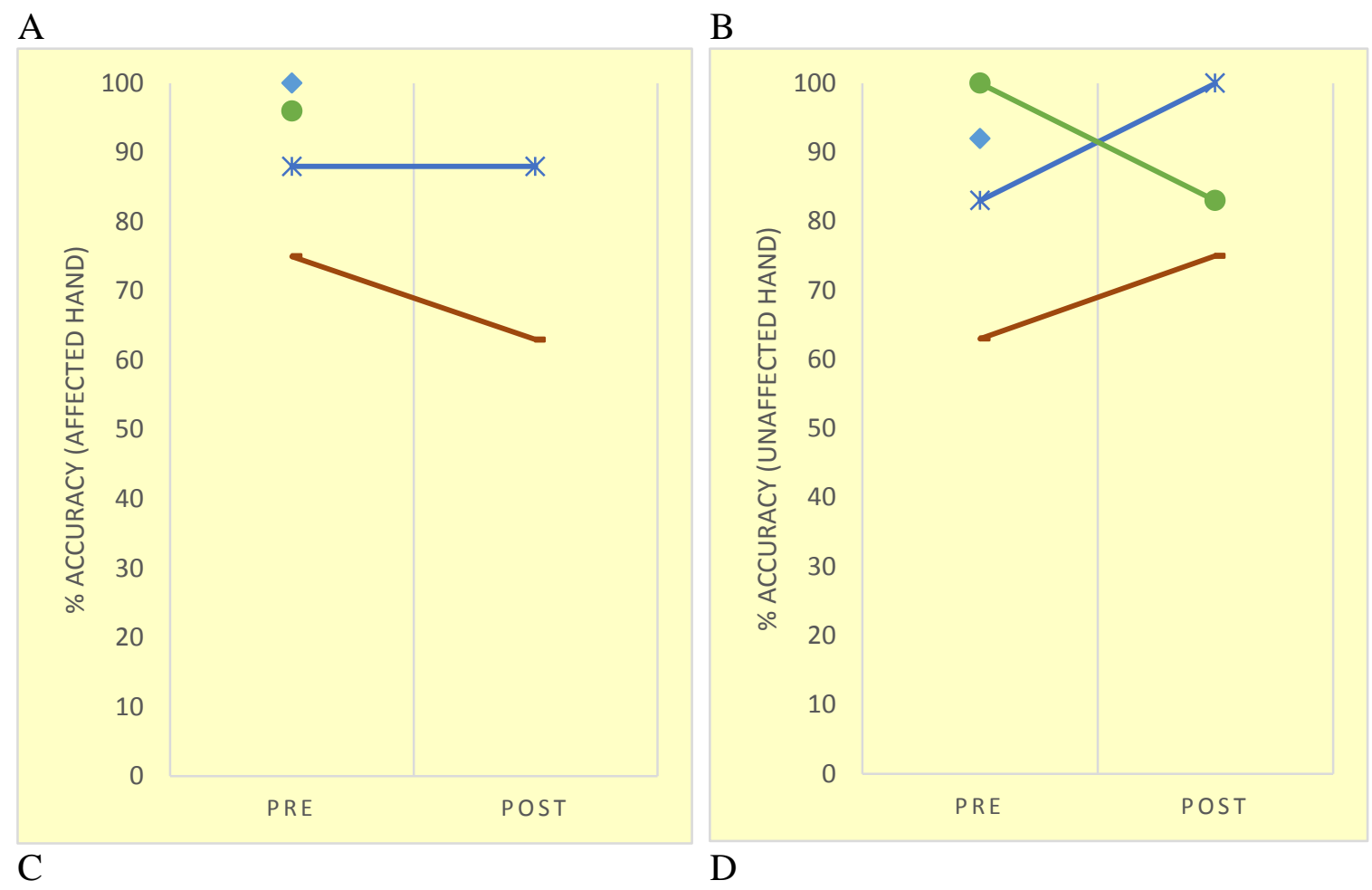




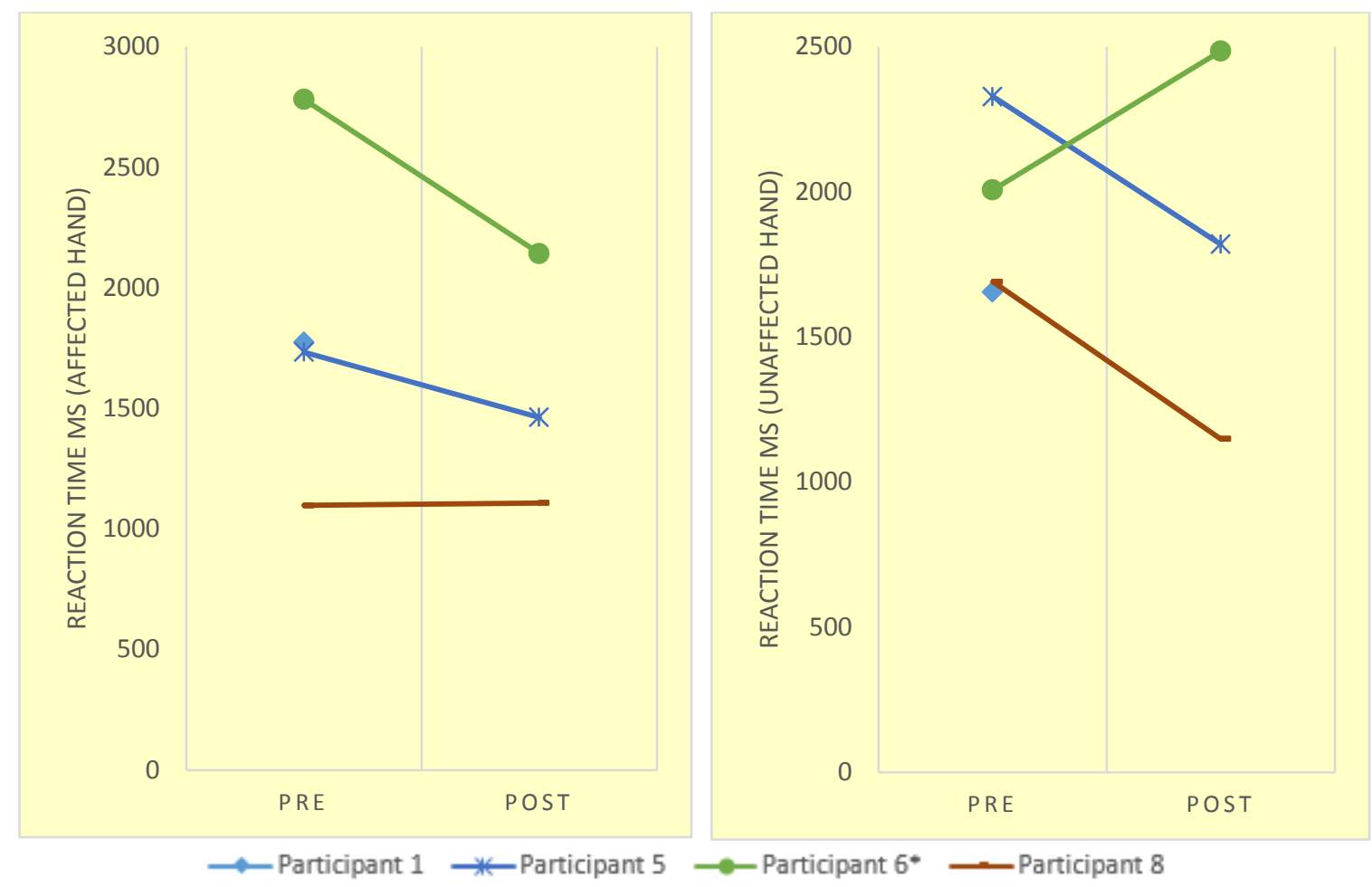

Figure Legend 3A-D: Data are presented for each individual participant. * indicates the participant received the placebo intervention. 
Figure 3A-B: Pain and TENS usage diary

(A)

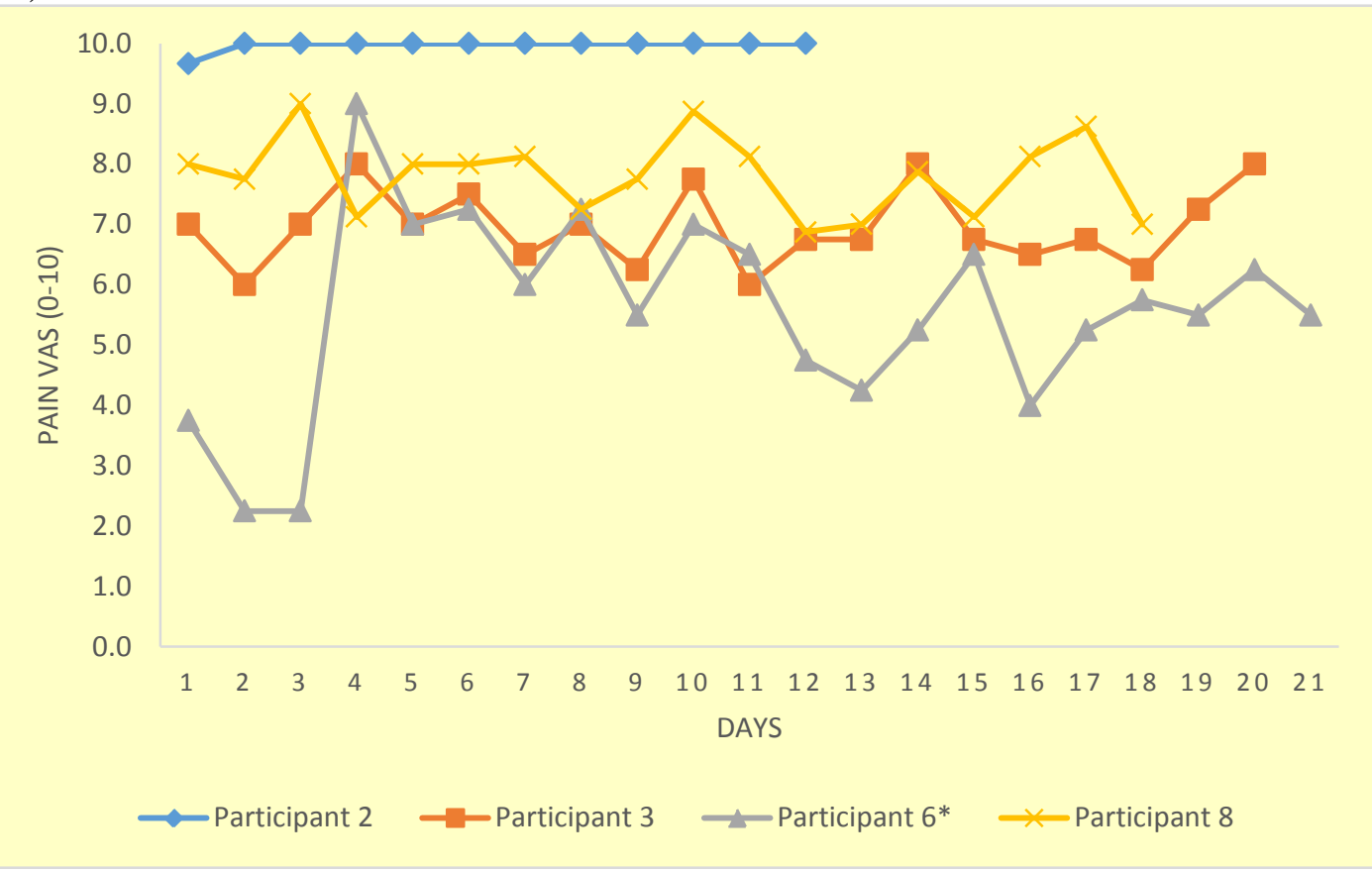


(B)

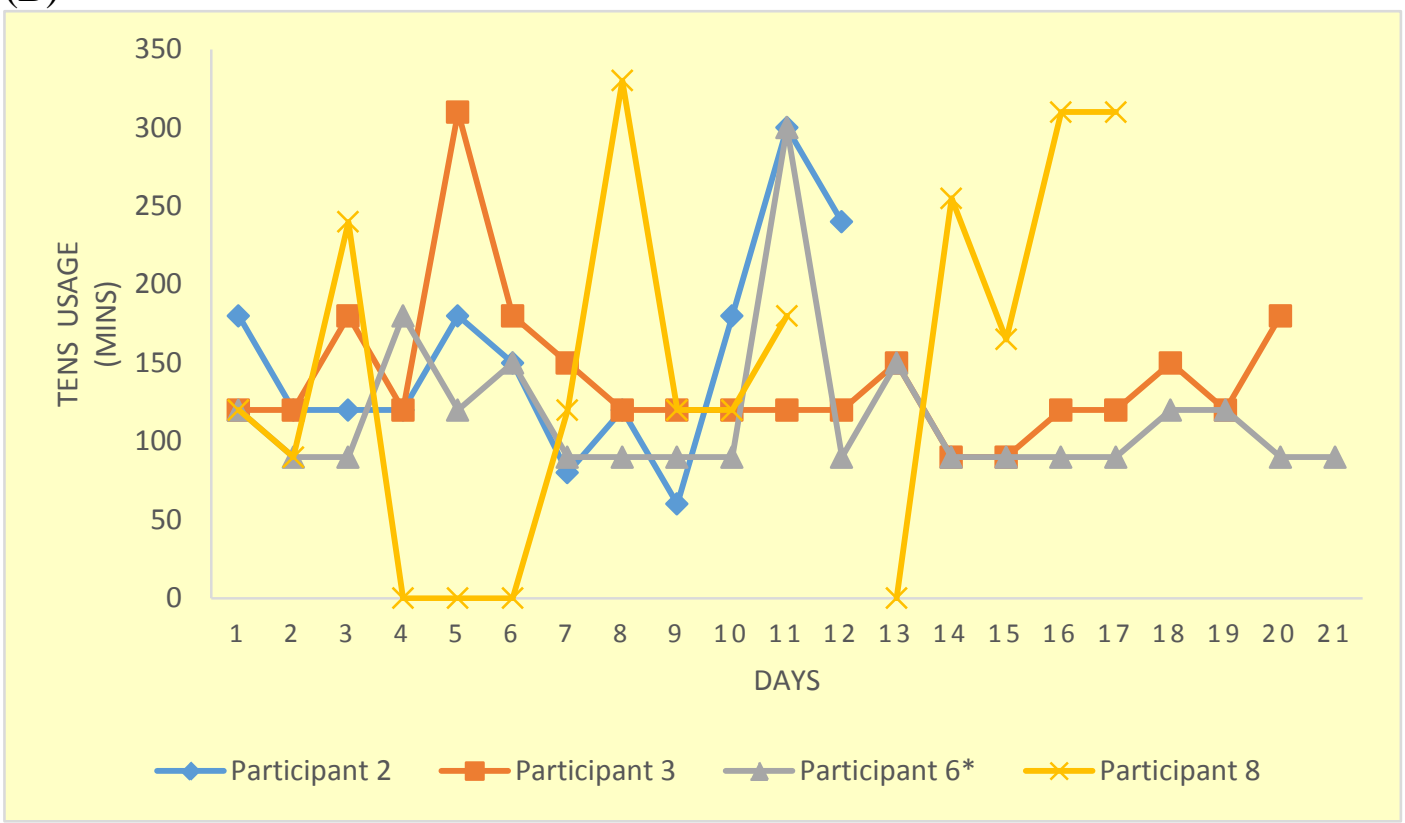

Figure Legend 3A-B: The top graph (A) shows the average pain daily pain reported by each participant that completed a diary. The bottom graph (B) shows the total TENS usage time each day for each participant that completed at diary. Missing data was left blank. *indicates that participant 6 was in the placebo group while participants 2, 3 and 8 were in the intervention group. 